\title{
AN ALTERNATE INTERPRETATION OF THE OORT CLOUD OF COMETS?
}

\author{
L. KRESAK
}

Some problems of the current interpretation of the oort cloud are discussed. If observational selection is taken into account, no significant difference in physical characteristics of old and new comets is apparent, in spite of the required change in the radiation mechanism after the first passage near the sun. The abundance of new comets puts special requirements on the relative size of the perihelion distances at which they lose their orbital characteristics and original surface properties, respectively. Stellar perturbations do not seem to be effective enough to displace the perihelia of new comets in a single revolution from the zone of insignificant planetary perturbations into the zone of detectability. Therefore, many physically new comets should appear as dynamically old, which is at variance with observational evidence. It is speculated whether the oort cloud really represents a reservoir of comets passing near the Sun for the first time or, alternatively, a region where the capability of building up extensive comas is being restored within periods of the order of $10^{6}$ to $10^{7}$ years.

The dynamical evidence of the Oort Cloud - clustering of reciprocal semimajor axes of comets within a narrow range around $4 \times 10^{-5} \mathrm{Al}^{-1}$ - is known to have important implications for the physico-chemical evolution of comets. It is believed that these "new" comets do not only approach the Sun for the first time in their history but also mostly only once as observable objects. The former conclusion is based on the fact that just a single passage through the planetary zone would smear out the observed peak in the distribution of $1 /$ a by random perturbations. The latter conclusion also seems to be inevitable, since otherwise the peak would be swamped by the abundant background of "old" comets experiencing second and subsequent passages. So, in addition to a continuous supply of new comets from the Oort cloud, it is required that almost all of them vanish, or at least dim appreciably, after the first approach to the Sun. At present we know orbits of 100 long-period comets with a reasonable accuracy in $1 / a$ (these orbits are based on observations extending over more than six months, with planetary perturbations duly taken into account and extrapolated backwards). Exactly one half of these comets can be classified as "new" and the other half as "old" in Dort's sense. So we have a suitable pair of statistical samples for intercomparison of their properties. One would expect to find significant differences associated with the drastic dimming between the first and second passage. The differences should be even more pronounced if the 
lack of old comets were due to a depletion mechanism allowing only a specific class of objects survive.

In fact, a number of distinctions of new comets from old ones have been suggested, such as higher absolute brightness, slower changes in brightness with distance from the Sun, asymmetric light curves (fainter after perihelion), prevalence of continuous spectra and dust tails, or tendency to outbursts and splitting. Surprising as it may appear, none of these effects could be identified as statistically significant in our samples. It seems that positive results of some previous investigations were due to a comparison of the comets classified as new with all the others. However, it requires a longer duration of positional observations to be sure that the comet is a new one. And in turn, longer observability is concomitant with photometric characteristics such as a high absolute brightness and a low photometric exponent, and lends more chance for observing such events as bursts or splitting. It is unfortunately impossible to determine the proportion of old and new comets among the inadequately observed objects in nearly parabolic orbits. But if a selection is made according to the duration of observation and reliability of classification, as done in our case, the differences in behaviour of the two groups definitely tend to disappear. There is also no evidence of the rapid fading of new comets starting already during their first passage near the Sun.

A lack of distinction is especially noteworthy as regards the perihelion distances. The median value, $q=1.6$, is exceeded by 23 old and 27 new comets a uniformity above mathematical expectation. There is some excess of new comets both at the smallest and largest values of $\mathrm{q}$ (below 0.4 and over $4 \mathrm{AU}$ ) but even this is on the margin of statistical significance.

The distribution of perihelion distances is of particular interest because it is required that the new comets have never before passed within some $20 \mathrm{AU}$ of the Sun. Otherwise the dispersion in $1 / a$ by planetary perturbations would be greater than the width of the peak of the observed distribution $\left( \pm 2 \times 10^{-5} \mathrm{AU}^{-1}\right)$, and the comets would appear as dynamically old. Only very large changes in perihelion distance by stellar perturbations would allow the new comets to enter the planetary zone and the region of observability during the same revolution around the Sun. This seemed to be possible with the original Oort's (1950) estimate of the radius of the cloud, $200,000 \mathrm{AU}$ and with the estimate of the diffusion rates by Shtejns and Sture (1962).

However, it was recently shown by Marsden et al. (1973) that the actual size of the cloud is probably much smaller. Comets of larger perihelion distance which are believed to yield the most reliable data due to weak nongravitational forces, display a remarkable concentration of aphelia near 50,000 AU from the Sun (Marsden and Sekanina 1973). There is also a number of comets with welldetermined orbits of original aphelion distance 20,000 to $30,000 \mathrm{AU}$ (1886 IX, 1890 II, 1941 VIII, 1954 V, 1959 I, 1959 IX, 1972 IX). A correction for nongravitational effects would tend to make these distances even somewhat smaller.

At the same time the recent results of Rickman (1976) indicate a lower efficiency of stellar perturbations. This would make the changes in perihelion distance one order of magnitude too small for $Q=40,000$ to $50,000 \mathrm{Ai}$, and two orders of magnitude too small for $Q=20,000$ to $30,000 \mathrm{AU}$. The discrepancy is smaller, but still significant, if the data of Shtejns and Sture are used. Accordingly, most of the new comets should lose their characteristic values of $1 / a$ before they make their first apparition.

To overcome this contradiction one would have to assume that the comets lose their capability of becoming bright in the same distant region where planetary perturbations become appreciable, i.e., some tens of AU from the Sun. Most comets which traverse this region stepwise in several revolutions (in a random walk allowing for temporary increases and decreases of q) would then never become visible, even if their perihelion distance ultimately becomes small enough. Those which become visible would represent exceptional objects which have under- 
gone very strong perturbations by stars passing nearby. The possibility that the observed concentration of new comets might be due to a perturbation by a single star that passed near the Sun 2 million years ago was already pointed out. by Marsden and Sekanina (1973). But once we assume that the new comets are due to a single stellar encounter, there is in fact no need for worrying about their fading because their present flux would not be concomitant with that of the old comets. Unfortunately, the distribution of the directions of the aphelia of new comets lends to supporting evidence for this explanation.

If we question one of the tacit assumptions of the current theories of cometary evolution - the continuous influx of new comets from the Oort ciloud we should do this with another point as well. All previous considerations relied on the assumption that the abrupt fading of new comets results from their exposure to solar radiation during the first passage near the Sun. This process is generally believed to be irreversible, as it probably involves a complete loss of the highly volatile materials deposited on the surface of the cometary nucleus since its formation. As an alternative we can inquire whether a long stay at the outskirts of the solar system cannot he the decisive point. If this could help to restore the original properties of the nuclear surface, then many comets classified as "new" should be properly called "renewed" or "rejuvenated." In principle, three mechanisms of rejuvenation are possible:

(a) accretion of a new active cover from outside,

(b) replacement of the lost surface layer by a transport of active material from inside, and

(c) destruction of an insulating crust formed on the surface of a deep, more volatile layer during the first apparition.

We have no supporting evidence of any of these mechanisms being actually present and effective enough. However, it is clear that the explanation in terms of rejuvenation would remove the difficulties with the rate of change of the perihelion distances because the brightness would depend solely on the aphelion distance of the preceding revolution. At the same time, a smaller supply of new comets and less drastic changes after the first passage near the Sun would be needed.

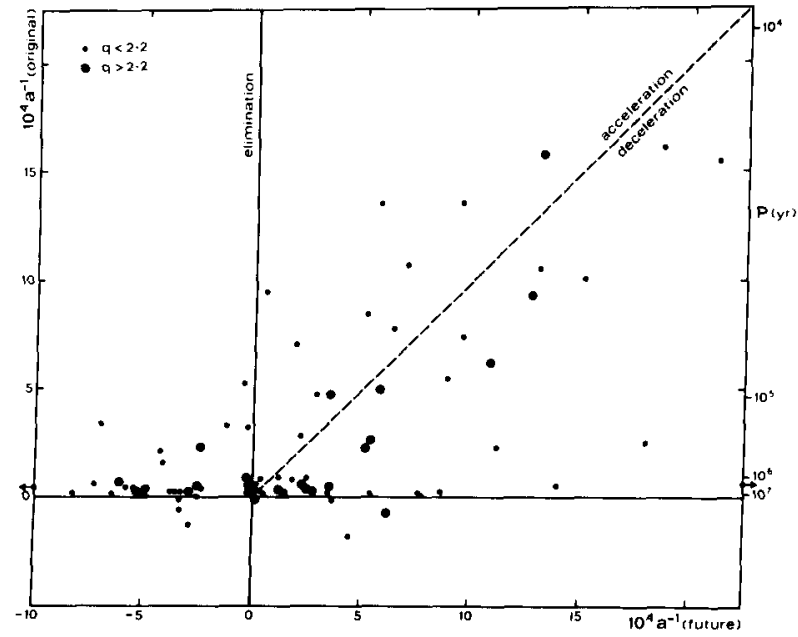

Figure 1. Original and future reciprocal semi-major axes of long-period comets with well determined orbits. Comets of perihelion distances larger than 2.2 AU (relatively free of non-gravitational effects) are distinguished by larger dots. The horizontal chain of new comets is seen to dissipate entirely by diffusion of their orbital energy. 
The situation is illustrated by Figure 1 showing the original and future reciprocal semimajor axes of long-period comets with well determined orbits. The osculating elements are taken from Marsden's Catalogue (1975), the perturbations from the list of Everhart and Reghavan (1970) and its supplements (Marsden's Comet Reports in QJRAS). Comets of larger perihelion distance, the orbits of which should be relatively free of nongravitational effects (Marsden and Sekanina 1973) are distinguished by larger dots. The horizontal chain of new comets is seen to dissipate entirely in the other coordinate. But always a fraction of the "new" comets retain, by an interplay of planetary perturbations, positions of their aphelia within the Oort Cloud, and a fraction of "old" comets reacquire, by a balance with the perturbations experienced in previous passages through the planetary zone, orbits characteristic for "new" comets. Apparently, a quasisteady state may arise, in which the observed overabundance of comets in the critical range of $1 / a$ is the result of a higher brightness of those bodies which have spent a longer time at a large distance from the Sun. The inner limit of the observed concentration in 1/a would be controlled by the efficiency of the rejuvenation process, the outer limit by the decrease of the flux due to longer revolution periods.

It is difficult to suggest any particular mechanism for this hypothetical process. However, its conditions can be specified by a minimum duration of $10^{6}$ years. I do by no means claim that this interpretation of the Oort Cloud changing it from a reservoir of new comets into a region of rejuvenation of old comets - is the correct one. It is just a matter of speculation. However, unless the inconsistencies of the present ideas on the rapid fading of new comets are removed, any alternate interpretation seems to deserve further investigation.

\section{REFERENCES}

Everhart, E., and Raghavan, N. 1970, Astron. J., 75, 258.

Marsden, B. G. 1975, Catalogue of Cometary orbits, Centr. Bur. Telegr. Astron., Cambridge, Mass.

Marsden, B. G., and Sekanina, Z. 1973, Astron. J., 78, 1118.

Marsden, B. G., Sekanina, Z., and Yeomans, D. K. 1973, Astron. J., 78, 211.

Oort, J. H. 1950, Bu11. Astron. Inst. Netherlands, 11, 91.

Rickman, H. 1976, Bul1. Astron. Inst. Czech., 27, 92.

Shtejns, K. A., and Sture, S. Ya. 1962, Astron. Zh., 39, 506.

\section{DISCUSSION}

ARNOLD: Can you give us the formal dynamical definition of "new" and "old" long-period comets?

KRESAK: The dividing line is at $10^{-4} \mathrm{AU}^{-1}$ in 1 /a where the high rate of occurrence of "new" comets steeply drops to the low background level of "old" comets.

WEISSMAN: In following long period comets with a Monte Carlo calculation it is found that about $10 \%$ return to the oort cloud at sometime in their lifetime. once there is most likely that stellar perturbations will raise their perihelia and remove them from the visible region among the inner planets.

KRESAK: The dynamical losses would be just the same as in the concept of rapid dimming of new comets. The point is that the distribution in 1/a would be affected by the rejuvenation process in such a way that the comets of longest revolution periods would reach again a high brightness irrespective of their previous orbital evolution. 
CHAPMAN: I know you do not claim to have identified any specific rejuvenation process. But there seem to be physical difficulties in forming a rejuvenated layer of material of any appreciable depth. Rejuvenation from within would seem to require a substantial energy source since a comet nucleus should be quite "dead" at cold temperatures in interstellar space. Certainly there are observational constraints on the volume density of material available to accrete onto a comet in $10^{6}$ years. What depth is required for the rejuvenated layer?

KRESAK: This would depend on the properties of the original surface which are still unknown; perhaps a depth of a few meters is a reasonable order-of-magnitude estimate for the layer removed at one apparition. The time scale of $10^{6}$ years is meant as an absolute lower limit because the mean revolution period of "new" comets is about $4 \times 10^{6}$ years, and the two approaches to the sun need not occur in two immediately following revolutions.

ANDERS: One immediate objection against your rejuvenation mechanism is the elemental composition of galactic cosmic rays. From the abundances of $L i, B e$, $B$ etc., Simpson and others have concluded that cosmic rays have traversed no more than $3-4 \mathrm{~g} / \mathrm{cm}^{2}$ of matter since they left their sources. Even if all this matter were located in the oort cloud, it could not build up meter-thick deposits in a few revolutions, as required by your rejuvenation mechanism.

KRESAK: The thickness of several meters is based on current estimates of the mass loss of comets per revolution. However, a rejuvenation process would not necessarily require a full replacement of the lost material by new deposits; changes in a much thinner surface coating might be sufficient. With a wide range of perihelion distances of the new comets it is difficult to understand why they all should lose their active layers just at the end of the first passage near the Sun. And we have no observational evidence of a drop in their light intensity during their first apparition. 